\title{
Texture Control for Improving Deep Drawability in Rolled and Annealed Aluminum Alloy Sheets
}

\author{
Hirofumi Inoue* and Takayuki Takasugi \\ Department of Materials Science, Graduate School of Engineering, Osaka Prefecture University, Sakai 599-8531, Japan
}

In order to find a possibility of texture control for improving deep drawability in rolled and annealed aluminum alloys, the relation among recrystallization texture, $r$-value and limiting drawing ratio was examined for sheet materials with various textures. By using specimens with $\{111\}$ texture prepared artificially, limiting drawing ratio could be measured in a wide range of average $r$-value from 0.4 to 1.6 . Experimental results demonstrated that there was a positive correlation between average $r$-value and limiting drawing ratio even in aluminum alloys. This means that an increase in average $r$-value leads to improvement of deep drawability. Warm rolling that forms shear texture including $\{111\}$ components, therefore, was conducted to enhance average $r$-value for Al-Mg and Al-Mg-Si alloys. Recrystallization texture of an annealed Al$\mathrm{Mg}$ alloy consisted of retained shear texture components in the surface layer and cube plus R orientations in the center layer. The average $r$-value was considerably improved compared with that of a cold rolled sheet. On the other hand, a T4-treated Al-Mg-Si alloy had a relatively weak cube texture on the whole, though the surface layer showed a different texture from the center. In this case, warm rolling was ineffective in improving average $r$-value, in spite of the existence of surface texture with higher $r$-value. However, the relation between recrystallization texture and experimental $r$-value was successfully explained for the Al-Mg-Si alloy as well as for the Al-Mg alloy, based on $r$-value calculations from overall texture through sheet thickness. [doi:10.2320/matertrans.L-MRA2007871]

(Received March 26, 2007; Accepted May 31, 2007; Published July 19, 2007)

Keywords: recrystallization texture, r-value, limiting drawing ratio, warm rolling, texture gradient

\section{Introduction}

In recent years, a trend of weight reduction in automobiles is rising from the viewpoint of reducing fuel consumption and exhaust gas emission. Above all, the application of aluminum alloys to automotive body panels is positively considered as a means of effective weight reduction. If aluminum alloy sheets are actually used for automotive body panels, the evaluation of press formability is required in addition to mechanical properties such as strength and ductility. In sheet steels, it is well known that limiting drawing ratio (LDR) evaluating deep drawability is greatly influenced by the Lankford value ${ }^{1)}$ expressed by a ratio of true strain in width to true strain in thickness, i.e. $r$-value $\left(r=\mathrm{d} \varepsilon_{w} / \mathrm{d} \varepsilon_{t}\right)$. Similarly, aluminum alloy sheets also seem to show a positive correlation between average $r$-value and LDR under limited conditions. ${ }^{2)}$ Here, when $r_{0}, r_{45}$ and $r_{90}$ are $r$-values at directions of 0,45 and $90^{\circ}$ to the rolling direction respectively, the average $r$-value is given by $\bar{r}=$ $\left(r_{0}+2 r_{45}+r_{90}\right) / 4$ conventionally. However, such a correlation has not been clear from experimental results so far, because the average $r$-value for aluminum alloys produced by conventional rolling and annealing processes generally shows rather lower values than 1 and exists only in a narrow range of 0.55 to 0.85 . 2,3 )

The $r$-value is closely related to crystallographic orientation. For polycrystalline materials, it is possible to increase the average $r$-value and/or the $r$-value at specific directions in a sheet plane by controlling crystallographic texture, which represents statistical distribution of grain orientations. The average $r$-value is much lower for aluminum alloy sheets manufactured by conventional cold-rolling and annealing processes than for commercial steel sheets. Figure 1 shows planar anisotropy of the $r$-value calculated by Taylor full

*Corresponding author, E-mail: inoue@mtr.osakafu-u.ac.jp

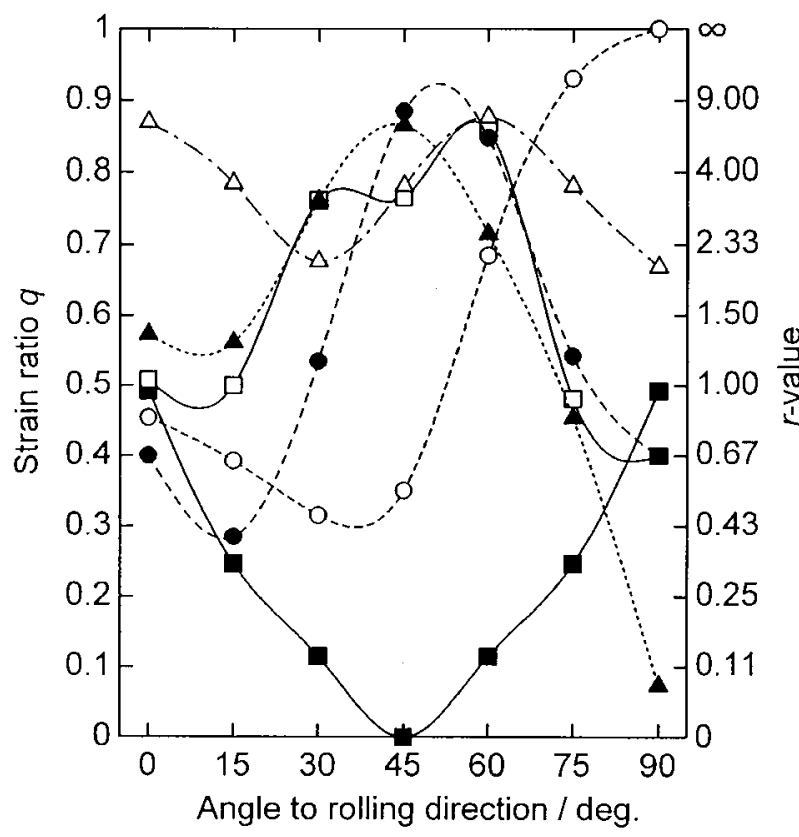

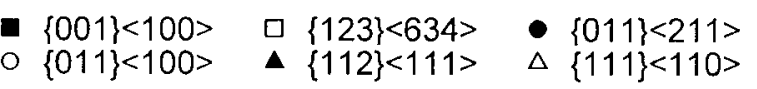

Fig. 1 Calculated $r$-values for representative ideal orientations with a Gaussian spread of $10^{\circ}$ as a function of angle to rolling direction. Strain ratio $q$ is a ratio of true strain in width to true strain in length: $q=$ $r /(1+r)$.

constraints model ${ }^{4)}$ for some ideal orientations with Gaussian distribution. ${ }^{5)}$ From this figure, it is suggested that there are the following two possible ways to achieve a high average $r$ value. One is to retain $\beta$-fiber rolling texture components such as $\{123\}\langle 634\rangle$ (S) and $\{011\}\langle 211\rangle$ (Bs) by pinning subgrains and recrystallized grains by fine particles precipitated during recovery and in the early stage of recrystallization in an annealing process. ${ }^{5,6)}$ The other is to evolve a $\{111\}$ 
component such as $\{111\}\langle 110\rangle$ during annealing from shear texture formed by warm rolling ${ }^{7,8}$ or differential speed rolling (asymmetric rolling), ${ }^{9,10)}$ which generally consists of major $\langle 110\rangle / / \mathrm{RD}$ fiber and minor $\langle 111\rangle / / \mathrm{ND}$ fiber. In the former case, the retained $\beta$-fiber texture components probably result in anisotropic $r$-values, which are high at a direction of $45^{\circ}$ to the rolling direction but low at directions of 0 and $90^{\circ}$, as shown in $\mathrm{S}$ and Bs orientations. In the latter case, the $r$-values of $\{001\}\langle 110\rangle$ and $\{112\}\langle 110\rangle$ orientations in shear texture result in strong planar anisotropy, as expected from those of $\{001\}\langle 100\rangle$ (cube) and $\{112\}\langle 111\rangle(\mathrm{Cu})$ orientations in Fig. 1, respectively. Consequently the $\{111\}\langle u v w\rangle$ orientations with high and isotropic $r$-value must be developed during annealing at the expense of other shear texture components.

There are numerous studies on processing and texture control for increasing $r$-value, while there are few reports on the correlation between $r$-value and LDR in aluminum and its alloy sheets. ${ }^{2}$ ) In the present study, first the correlation between $r$-value and LDR was examined in a wide range of average $r$-value (0.4-1.6), using not only the cold rolled and annealed sheets with different recrystallization textures but also the artificially prepared specimens with a $\{111\}$ preferred orientation, which is hardly developed in fcc metals. After a positive correlation between average $r$-value and LDR was verified, the possibility of texture control by warm rolling was examined for $\mathrm{Al}-\mathrm{Mg}$ and $\mathrm{Al}-\mathrm{Mg}-\mathrm{Si}$ alloys in order to increase average $r$-value with small anisotropy.

\section{Experimental Procedure}

The as-received materials mainly employed for examination of the correlation between average $r$-value and LDR were $8 \mathrm{~mm}$ thick hot rolled $\mathrm{Al}-4.3$ mass $\% \mathrm{Mg}$ alloy plates containing $0,0.1,0.3$ and 0.8 mass $\%$ Fe. After solution treatment was performed under various conditions depending on $\mathrm{Fe}$ content $(693 \mathrm{~K}-3.6 \mathrm{ks}$ for $0 \% \mathrm{Fe}, 793 \mathrm{~K}-3.6 \mathrm{ks}$ for $0.1 \% \mathrm{Fe}, 793 \mathrm{~K}-10.8 \mathrm{ks}$ for 0.3 and $0.8 \% \mathrm{Fe}$ ), these plates were cold rolled to $90 \%$ reduction in thickness. Then the cold rolled sheets were annealed at $573 \mathrm{~K}$ for appropriate times followed by water quenching to obtain fully recrystallized microstructure. A commercial purity aluminum AA1100 plate of $11 \mathrm{~mm}$ in thickness, which was hot rolled at $803 \mathrm{~K}$ to 97\% reduction, was used for intentionally making sliced specimens with a $\{111\}$ texture as a main component. In other words, the AA1100 plate with $\beta$-fiber rolling texture was sliced at an oblique plane rotated by $22^{\circ}$ from the rolling plane around the transverse direction, as shown in Fig. 2. Accordingly the sliced specimens ought to exhibit a high pole density at the center of $\{111\}$ pole figure at this stage. These were finally annealed at $623 \mathrm{~K}$ for $300 \mathrm{~s}$ followed by water quenching to obtain equiaxed recrystallized grains. A hot extruded AA6061 alloy round bar of $88 \mathrm{~mm}$ in diameter was also used for intentionally making sliced specimens with a $\{111\}$ texture. That is to say, the round bar with $\langle 111\rangle$ plus $\langle 100\rangle$ double fiber texture, which was produced by hot extrusion at $803 \mathrm{~K}$ followed by immediate water-shower cooling, was sliced at a cross section perpendicular to the extrusion direction, as shown in Fig. 3. The sliced specimens were finally $\mathrm{T} 4$-treated, namely, solution treated at $803 \mathrm{~K}$ for

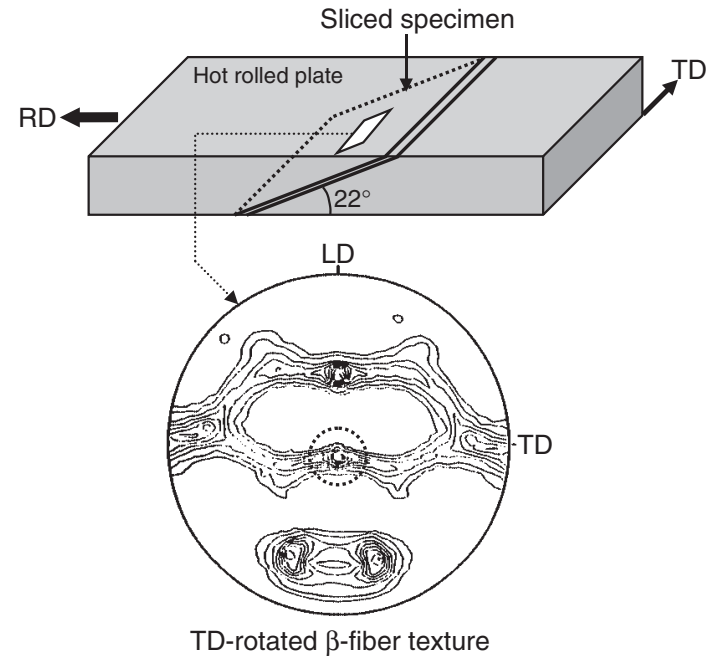

Fig. 2 Schematic illustration showing a method of preparing specimen with $\{111\}$ texture from hot rolled thick plate and $\{111\}$ pole figure for the sliced AA1 100 specimen annealed at $623 \mathrm{~K}$ for $300 \mathrm{~s}$. Contour levels: 1,2 , $3,4,5,6,7,8,9,10$.
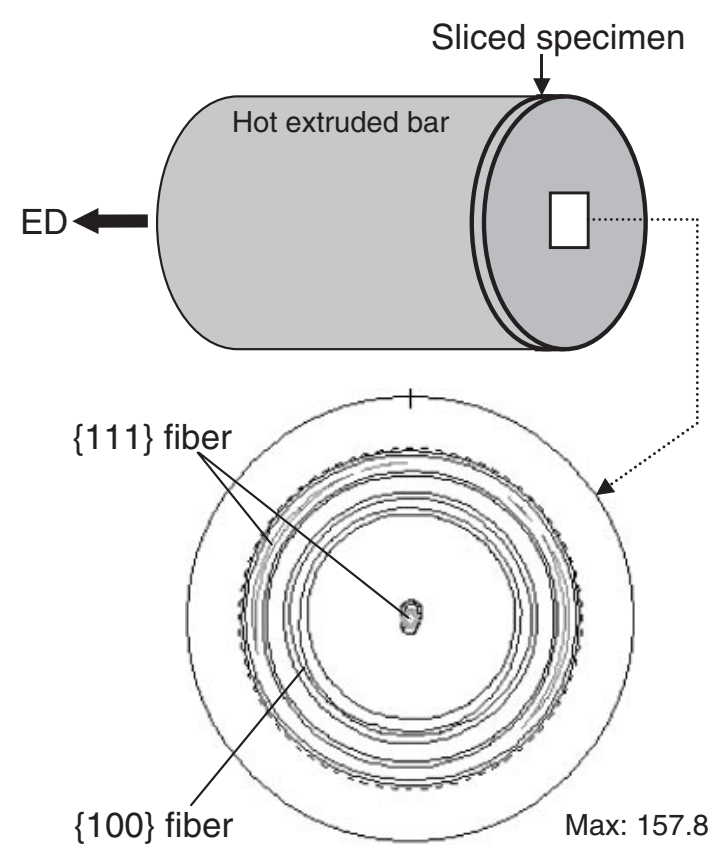

Fig. 3 Schematic illustration showing a method of preparing specimen with $\{111\}$ texture from hot extruded bar and $\{111\}$ pole figure for the sliced AA6061 specimen solution treated at $803 \mathrm{~K}$ for $1.2 \mathrm{ks}$ and subsequently aged at room temperature for more than 4 days. Contour levels: $1,2,4,8,16,32,64,128$.

$1.2 \mathrm{ks}$ followed by water quenching and subsequently aged at room temperature for more than 4 days, in order to obtain a recrystallized state and enhance ductility.

The as-received materials employed for examination of texture control by warm rolling were a hot rolled Al4.3 mass $\% \mathrm{Mg}-0.3$ mass $\% \mathrm{Fe}$ alloy plate of $8 \mathrm{~mm}$ in thickness and a hot rolled AA6016 (Al-0.6 mass $\% \mathrm{Mg}-1.0$ mass $\% \mathrm{Si}$ ) plate of $9 \mathrm{~mm}$ in thickness. These plates were annealed at $823 \mathrm{~K}$ for $1.8 \mathrm{ks}$ in an $\mathrm{Al}-\mathrm{Mg}-0.3 \% \mathrm{Fe}$ alloy and at $813 \mathrm{~K}$ for $3.6 \mathrm{ks}$ in AA6016 to obtain recrystallized microstructure and dissolve as many precipitates as possible. Then the Al-Mg- 
Table 1 Orientation density for preferred orientations of recrystallization texture in Fe-added Al-4.3 mass $\% \mathrm{Mg}$ alloy sheets cold rolled to $90 \%$ reduction and annealed at $573 \mathrm{~K}$. The numerical values denote intensity ratio of preferred orientation to random orientation.

\begin{tabular}{lccc}
\hline Orientation & Al-Mg & Al-Mg-0.1\%Fe & Al-Mg-0.3\%Fe \\
\hline Cube $\{001\}\langle 100\rangle$ & 41.2 & 17.9 & 20.5 \\
$\mathrm{R}\{123\}\langle 634\rangle$ & 2.7 & 5.8 & 6.2 \\
\hline
\end{tabular}

$0.3 \% \mathrm{Fe}$ alloy was warm rolled to approximately $90 \%$ reduction by four passes without and with lubricant at a starting temperature of $623 \mathrm{~K}$ at rolling speeds of 1 and $0.417 \mathrm{~m} / \mathrm{s}$, respectively. For comparison, the $90 \%$ cold rolled sheet was also prepared at about $15 \%$ reduction per pass under lubrication. These rolled sheets were finally annealed at $573 \mathrm{~K}$ for $10 \mathrm{ks}$ followed by water quenching to obtain a fully recrystallized state. On the other hand, the AA6016 alloy was warm rolled to approximately $90 \%$ reduction by three passes without lubricant at a starting temperature of $673 \mathrm{~K}$. This alloy was also cold rolled to the same final gauge as the warm rolled sheet. The rolled AA6016 sheets were annealed (i.e. solution treated) at $793 \mathrm{~K}$ for $960 \mathrm{~s}$, immediately quenched in water and aged at room temperature for more than 4 days (T4-treatment).

To analyze textures of various materials described above, incomplete pole figures with a range of $\alpha=0$ to $75^{\circ}$ were measured for (111), (200), (220) and (311) diffraction planes by the Schulz reflection method using $\mathrm{CuK} \alpha$ radiation. Exceptionally the complete pole figures were determined by adding data with a range of $\alpha=55$ to $90^{\circ}$ measured by the Decker-Asp-Harker transmission method for AA1100 sliced specimens. Intensity correction of pole figures was made using a randomly oriented sample prepared by hot-pressing aluminum powder. From four pole figures, the crystallite orientation distribution function (ODF) including odd terms for ghost correction was calculated up to an expansion order of 22, using the "Standard ODF" software developed by the present author, based on the iterative series expansion method. ${ }^{11,12)}$ For some recrystallized materials, especially for warm rolled and annealed materials with texture gradient along the thickness direction, overall texture through sheet thickness was also analyzed from incomplete pole figures measured on the cross section perpendicular to the rolling direction (RD-section) by the Schulz reflection method, because $r$-value can be related to a statistically average texture through thickness. To prepare an RD-sectional sample for pole figure measurement with an area of $25 \mathrm{~mm} \times 25 \mathrm{~mm}$, a number of pieces with a size of $3 \mathrm{~mm}$ in the rolling direction (RD) and $25 \mathrm{~mm}$ in the transverse direction (TD) were cut out from the sheet material and then stacked and embedded in resin. The ODF on the RD-basis calculated from incomplete pole figures on the RD-section was transformed into the normal ODF on the ND-basis on the assumption of orthorhombic sample symmetry. ${ }^{13)}$ In addition, to observe a textural change through thickness, crystal orientation maps of annealed sheets were measured in the longitudinal section by means of the electron backscatter diffraction pattern (EBSD) method using a scanning electron microscope.

The $r$-values of annealed (or solution treated) sheets were determined from changes in gauge length and width with applying a tensile strain of $10 \%$, mainly using test pieces with gauge length of $10 \mathrm{~mm}$ and width of $8 \mathrm{~mm}$ cut out at seven directions of $0,15,30,45,60,75$ and $90^{\circ}$ to the rolling direction. In this case, the average $r$-value $\bar{r}$ can be defined by

$$
\bar{r}=\left(r_{0}+2 r_{15}+2 r_{30}+2 r_{45}+2 r_{60}+2 r_{75}+r_{90}\right) / 12 .
$$

To evaluate LDR of annealed (or solution treated) sheets, a deep drawing test was performed using a flat head punch of 10 or $33 \mathrm{~mm}$ in diameter depending on the size of sheet specimens. LDR was determined from a maximum blank diameter when deep drawing was successful.

Planar anisotropy of $r$-value was calculated from ODF of recrystallization texture using the method proposed by Bunge, ${ }^{14,15)}$ based on the Taylor full constraints (FC) model ${ }^{4)}$ and the relaxed constraints (RC) model $^{16)}$ relaxing some shear strain components from the fully constrained state, in order to relate the measured $r$-values at various directions to recrystallization texture.

\section{Results}

\subsection{Correlation between $r$-value and limiting drawing ratio}

\subsubsection{Texture and $r$-value}

Samples with different recrystallization textures were prepared to change the $r$-value. All the cold rolled Fe-added $\mathrm{Al}-4.3 \% \mathrm{Mg}$ alloys exhibited a pure metal type rolling texture consisting of $\beta$-fiber, namely $\mathrm{Cu}, \mathrm{S}$ and $\mathrm{Bs}$ orientations as main components in an as-rolled state, though the intensity was somewhat lower in a $0.8 \% \mathrm{Fe}$-added alloy than in other alloys. Table 1 shows a change in orientation density with $\mathrm{Fe}$ content for preferred orientations of recrystallization texture after annealing at $573 \mathrm{~K}$ for 3.5 and $10 \mathrm{ks}$ in Fe-free and Feadded alloys, respectively. Recrystallization texture of a Fefree alloy consists of a very strong cube orientation $\{001\}\langle 100\rangle$ and a rather weak $\mathrm{R}$ orientation $\{123\}\langle 634\rangle$, which is distinguished from $\mathrm{S}$ orientation in rolling texture, while Fe-added alloys show a decreased cube orientation and an increased $\mathrm{R}$ orientation. Although cube orientation is dominant for all alloys, it is obvious that Fe-addition reduces cube orientation drastically. This probably results from the suppression of growth of cube oriented grains by fine $\mathrm{Al}_{3} \mathrm{Fe}$ particles precipitated during recovery and/or in the early stage of recrystallization. The Fe-addition above $0.1 \%$, however, does not bring about a significant change in texture. This is attributable to little solubility of iron in aluminum at $573 \mathrm{~K}^{17)}$ Such textural change by Fe-addition reflects a remarkable change in $r$-value, as shown in Fig. 4. Since a Fe-free alloy had well-developed cube texture, the $r$-value shows a concave curve with a very low $r$-value of 0.24 at a direction of $45^{\circ}$ to $\mathrm{RD}$, as easily understood from Fig. 1. In contrast, $\mathrm{Fe}$-added alloys show relatively high $r$-values around the $45^{\circ}$ direction. Suppression of cube orientation and moderate development of $\mathrm{R}$ orientation, 


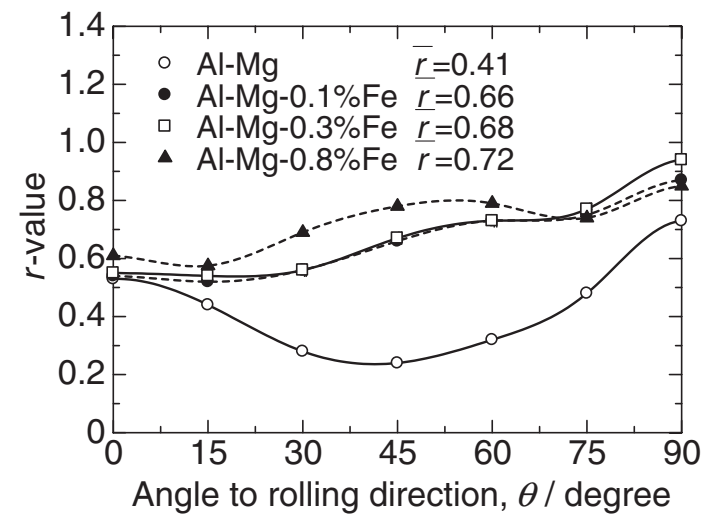

Fig. 4 Planar anisotropy of $r$-value for Fe-free and Fe-added Al-4.3\% $\mathrm{Mg}$ alloys cold rolled to $90 \%$ reduction in thickness and subsequently annealed at $573 \mathrm{~K}$ for 3.5 and $10 \mathrm{ks}$, respectively.

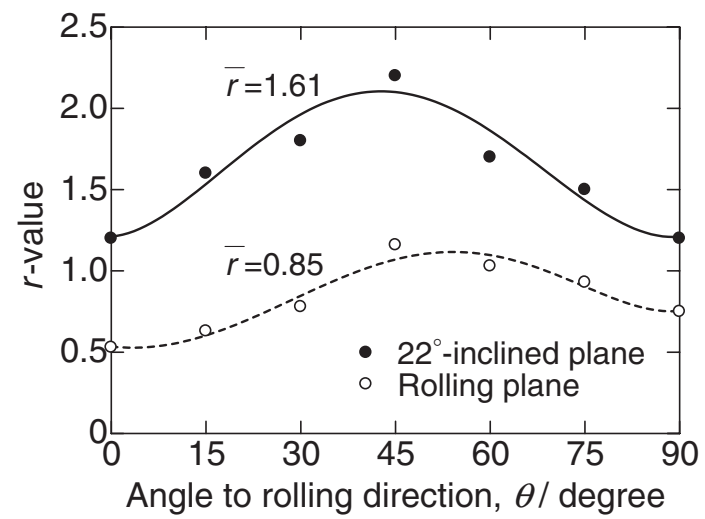

Fig. 5 Planar anisotropy of $r$-value for $22^{\circ}$-inclined plane and rolling plane of hot rolled AA1100 thick plate shown in Fig. 2.

which are caused by small Fe-addition, result in small anisotropy as a whole, because $\mathrm{R}$ orientation gives a fairly high $r$-value at the $45^{\circ}$ direction. Consequently, average $r$-value also increases from 0.41 in a Fe-free alloy to 0.66 in a $0.1 \%$ Fe-added alloy. Further Fe-addition above $0.1 \%$, however, causes only a small increase in average $r$-value.

A hot rolled AA1100 plate almost retained a strong $\beta$-fiber texture even after annealing at $623 \mathrm{~K}$ for $300 \mathrm{~s}$, although cube orientation was slightly developed. Naturally, the specimen sliced at an oblique plane of $22^{\circ}$ to the rolling plane from the same hot rolled plate had the $\beta$-fiber texture rotated by $22^{\circ}$ around TD-axis (TD-rotated $\beta$-fiber texture). As observed in a $\{111\}$ pole figure of Fig. 2 , the TD-rotated $\beta$-fiber texture, which included a $\{111\}$ component close to $\{111\}\langle 123\rangle$ orientation together with other orientations, was retained even in the sliced and annealed specimen. Planar anisotropy of $r$-value for these annealed materials is shown in Fig. 5. A hot rolled and annealed AA1100 plate has a high $r$-value of more than 1.0 at the $45^{\circ}$ direction and consequently the average $r$-value becomes higher than the cold rolled and annealed Al-Mg alloys. Such anisotropy results mainly from a retained $\beta$-fiber texture components such as $\mathrm{Cu}, \mathrm{S}$ and $\mathrm{Bs}$ orientations which show the highest $r$-value around the $45^{\circ}$ direction (see Fig. 1). On the other hand, the specimen inclined at $22^{\circ}$ to the rolling plane demonstrates a very high

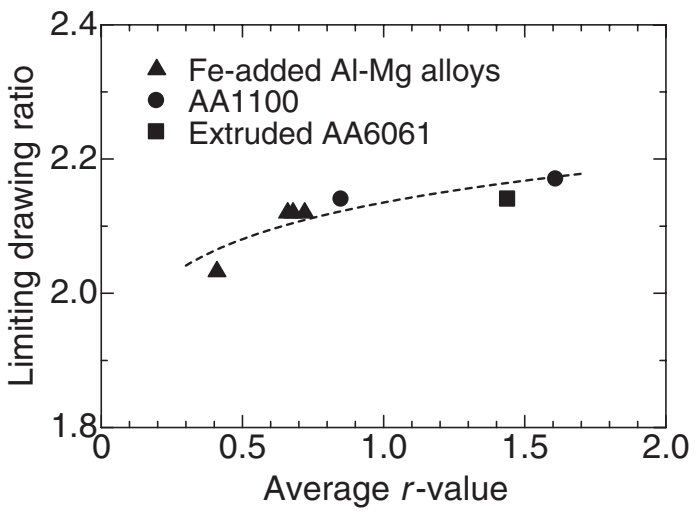

Fig. 6 Correlation between average $r$-value and limiting drawing ratio (LDR) for aluminum alloys with various textures.

average $r$-value of 1.61 in an annealed state. Surprisingly the $r$-value exceeds 1.2 at all directions. It has been proven that the existence of a $\{111\}$ orientation as a main component enables us to vastly improve average and minimum $r$-values in aluminum.

A hot extruded AA6061 round bar had a very strong $\langle 111\rangle+\langle 100\rangle$ double fiber texture along the extrusion direction (ED). This means that the specimen sliced at a cross section perpendicular to ED possesses a sharp $\{111\}+$ $\{100\}$ texture. The sliced specimen of an as-extruded material showed high $r$-values of about 1.34 at any direction because of a perfect fiber texture with annular distribution in the pole figure. However, since its microstructure consisted of elongated deformed grains, the correlation between $r$-value and LDR was evaluated for a T4-treated material that was in a fully recrystallized state. The T4-treated specimen retained a sharp $\{111\}+\{100\}$ texture (Fig. 3 ) and showed an $r$-value of 1.44 higher than that of the as-extruded specimen due to a slight increase of $\{111\}$ texture. The $r$-value, however, is not as high as expected, in spite of a very strong pole density over 150 at the center of $\{111\}$ pole figure. It seems that a strong $\{100\}$ texture canceled out $r$-value enhancement by a $\{111\}$ texture in some degree.

\subsubsection{Limiting drawing ratio}

It is not easy to relate texture to LDR quantitatively, but annealed specimens with a $\{111\}$ component tended to have a high LDR. In fact, the sliced specimen inclined at $22^{\circ}$ to the rolling plane had the highest average $r$-value and showed the highest LDR of 2.17 among all specimens. Figure 6 shows a correlation between average $r$-value and LDR for the specimens with various average $r$-values ranging from 0.41 to 1.61. This graph proves that LDR essentially increases with increasing average $r$-value, though there is some scatter of experimental data. This means that increasing average $r$ value by texture control leads to improving deep drawability of aluminum alloys. It has been suggested that a workhardening exponent, $n$-value also affects LDR. $^{2,18)}$ It is however noted that $n$-value of fully annealed aluminum alloys was in a relatively narrow range of 0.24 (AA6061) to 0.33 (Al-Mg alloy) and therefore hardly affected LDR, as inferred from theoretical calculation that showed an effect of $n$-value on LDR. ${ }^{18)}$ 

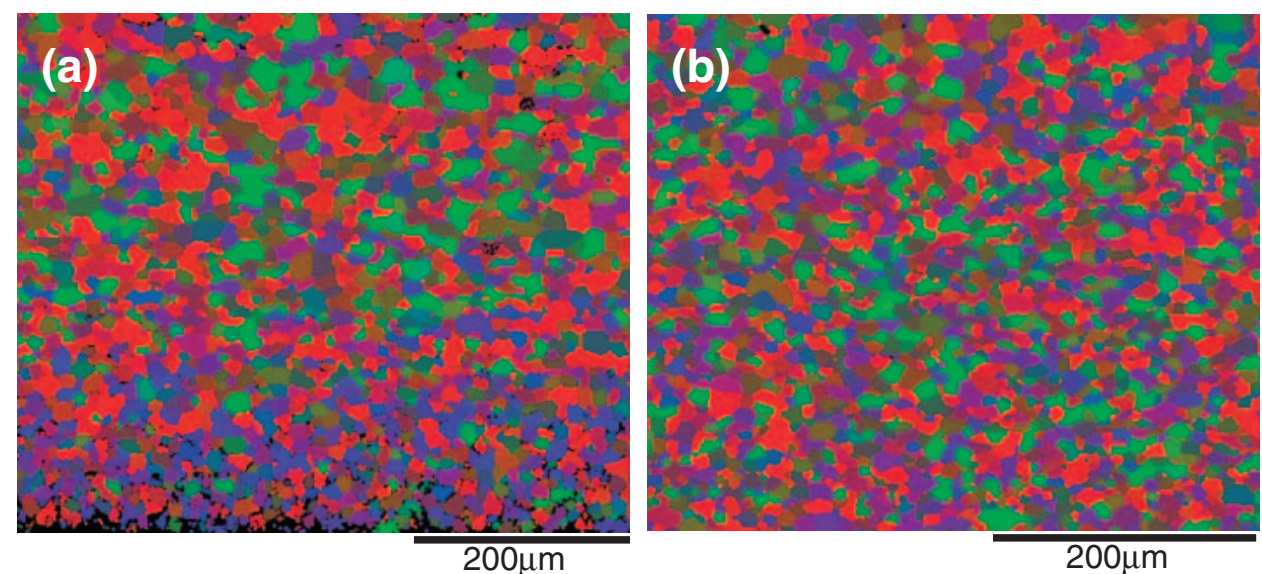

Center

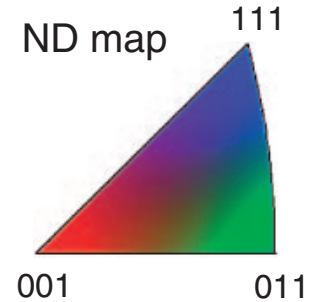

Surface

Fig. 7 Orientation maps in longitudinal section of Al-4.3\% Mg- $0.3 \%$ Fe alloy sheets, (a) warm rolled to $87 \%$ reduction at $623 \mathrm{~K}$ at rolling speed of $1 \mathrm{~m} / \mathrm{s}$ or (b) cold rolled to $90 \%$ reduction, and subsequently annealed at $573 \mathrm{~K}$ for $10 \mathrm{ks}$.

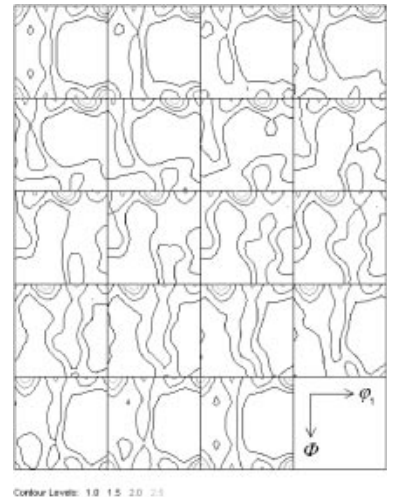

Warm rolled sample A

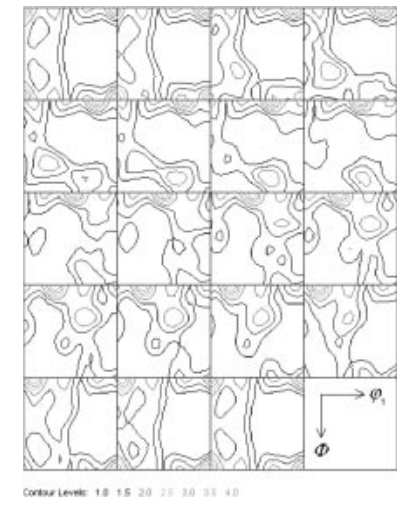

Warm rolled sample B

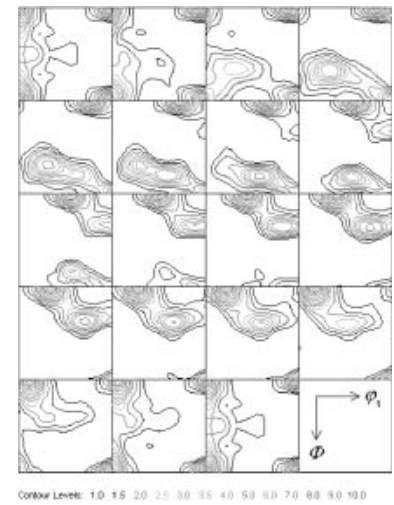

Cold rolled sample $\mathrm{C}$

Fig. 8 Overall textures through sheet thickness for Al-4.3\% Mg-0.3\%Fe alloy sheets, warm rolled to about $90 \%$ reduction at rolling speeds of $1 \mathrm{~m} / \mathrm{s}$ (sample A) and $0.417 \mathrm{~m} / \mathrm{s}$ (sample B) or cold rolled to $90 \%$ reduction (sample C), and subsequently annealed at $573 \mathrm{~K}$ for $10 \mathrm{ks}$. Contour levels: $1,1.5,2,2.5,3,3.5,4,5,6,7,8,9,10$.

\subsection{Texture control by warm rolling}

\subsubsection{Texture and $r$-value in Al-Mg alloy}

In aluminum alloys, warm rolling generally tends to develop shear texture consisting mainly of the $\langle 110\rangle / / \mathrm{RD}$ fiber ranging from $\{001\}\langle 110\rangle$ to $\{111\}\langle 110\rangle$ under the sheet surface due to friction with rolls. ${ }^{7,8)}$ However, shear texture cannot be deeply developed up to the middle of sheet thickness by normal warm rolling. It is important to develop shear texture from the surface to a deeper layer. Warm rolling with large reduction per pass may develop shear texture toward the center layer more deeply. Therefore, the reduction per pass was set above $50 \%$ based on a gap between rolls in the present study. In addition to the formation of shear texture extending toward the center layer, the effect of Fe-addition on recrystallization texture, which suppressed evolution of cube texture during annealing, can be also expected, especially in the vicinity of the center layer where $\beta$-fiber texture will be formed. In practice, as-rolled Fe-added $\mathrm{Al}-\mathrm{Mg}$ alloys showed a strong shear texture including a $\{111\}\langle 110\rangle$ orientation in the range from the surface to a quarter thickness and a strong $\beta$-fiber texture in the range from a quarter thickness to a half thickness, i.e. to the center of thickness. ${ }^{13)}$ Through-thickness orientation distribution after recrystallization of a warm rolled $\mathrm{Al}-4.3 \% \mathrm{Mg}-0.3 \% \mathrm{Fe}$ alloy sheet is shown in Fig. 7, compared with that of a cold rolled one. The $\{111\}$ oriented grains consisting mainly of $\{111\}\langle 110\rangle$ are observed at a high frequency in the range from the surface to a quarter thickness. In contrast, orientation distribution in the range from the quarter thickness to the center is composed of cube and retained $\beta$-fiber components, being similar to that in the whole range of a cold rolled and annealed sheet with relatively homogeneous texture. In order to relate recrystallization texture to $r$-value that is a property as the sheet material, overall textures through sheet thickness were analyzed in the Al-4.3\% Mg$0.3 \% \mathrm{Fe}$ alloy sheets warm or cold rolled and subsequently annealed. The results are shown in Fig. 8. The warm rolled sample A, which would be subject to the largest shear strain during rolling, shows a weak mixed texture derived from shear and $\beta$-fiber textures. The main components are $\{111\}\langle 110\rangle,\{001\}\langle 210\rangle$, cube and R orientations. On the other hand, the cold rolled sample $\mathrm{C}$, which would be deformed under plane strain during rolling, shows a relatively strong recrystallization texture with cube and $\mathrm{R}$ orientations. Texture of the warm rolled sample B is intermediate between the textures of two samples described above, but cube orientation is not strong so much.

Figure 9 shows planar anisotropy of $r$-value for these 

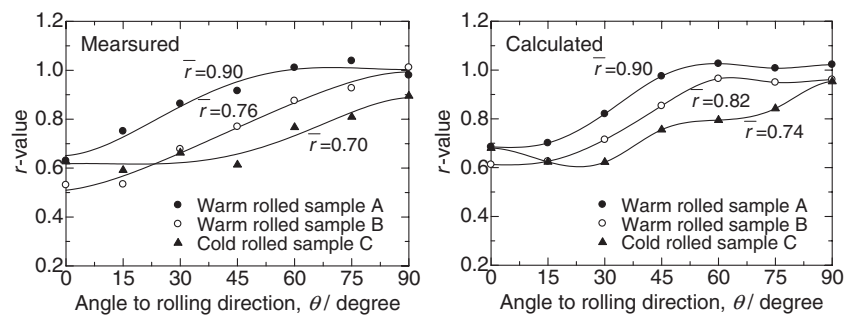

Fig. 9 Planar anisotropy of $r$-value measured and calculated by the relaxed constrains (RC) model from overall textures through sheet thickness for three samples shown in Fig. 8.

annealed samples. It is obvious from the measured values that the warm rolled sample A exhibits the highest $r$-values at almost all directions. As a result, its average $r$-value is improved with an increment of 0.2, compared with that of the cold rolled sample. The $r$-values calculated by the RC model from overall textures through thickness are, on the whole, in good agreement with the measured $r$-values. Here as the relaxed shear component in the calculation, only the shear strain perpendicular to the tensile direction was taken, because this model gave the better results than the FC model and the RC model based on other relaxed shear components. This means that for solid-solution hardenable Al-Mg alloys, the calculated $r$-values by the FC model assuming homogeneous deformation in all grains are not consistent with the measured $r$-values and that the shear strain perpendicular to the tensile direction hardly suffer constraint by chucks of a tensile testing machine. This result may be related to inhomogeneous deformation in $\mathrm{Al}-\mathrm{Mg}$ alloys, unlike commercial purity aluminum and $\mathrm{Al}-\mathrm{Mg}-\mathrm{Si}$ alloys.

\subsubsection{Texture and $r$-value in Al-Mg-Si alloy}

In this alloy as well as in the above Al-Mg alloy, warm rolling with large reduction per pass was performed to deeply develop shear texture. Figure 10 shows overall textures through sheet thickness for cold or warm rolled and T4treated AA6016 alloy, together with textures in the surface and center layers of a warm rolled and T4-treated sheet. Recrystallization texture of the cold rolled sample is composed of cube, $\{011\}\langle 233\rangle(\mathrm{P})$ and $\{011\}\langle 100\rangle$ (Goss) orientations that are generally observed in age-hardenable $\mathrm{Al}-\mathrm{Mg}-\mathrm{Si}$ alloys. ${ }^{19)}$ On the other hand, the warm rolled sample has a considerably weaker recrystallization texture but similar preferred orientations to the cold rolled one. This weak texture of the warm rolled sample results from the formation of a different texture near the surface, where near$\mathrm{R}$ and $\{001\}\langle 210\rangle$ orientations are observed as main components. These orientations seem to have been formed from shear texture by solution treatment at a high temperature of $793 \mathrm{~K}$. In contrast, recrystallization texture in the center layer of the warm rolled sample is similar to that of the cold rolled one, except for a weakened $\mathrm{P}$ orientation.

Figure 11 shows planar anisotropy of $r$-value measured and calculated by the FC model from the overall textures (Fig. 10(a) and (b)) for T4-treated AA6016 alloy sheets. In both samples, the calculated values are in good agreement with the measured values. Although the RC models with various relaxed shear strains were attempted, the FC model assuming homogeneous deformation in all grains gave the
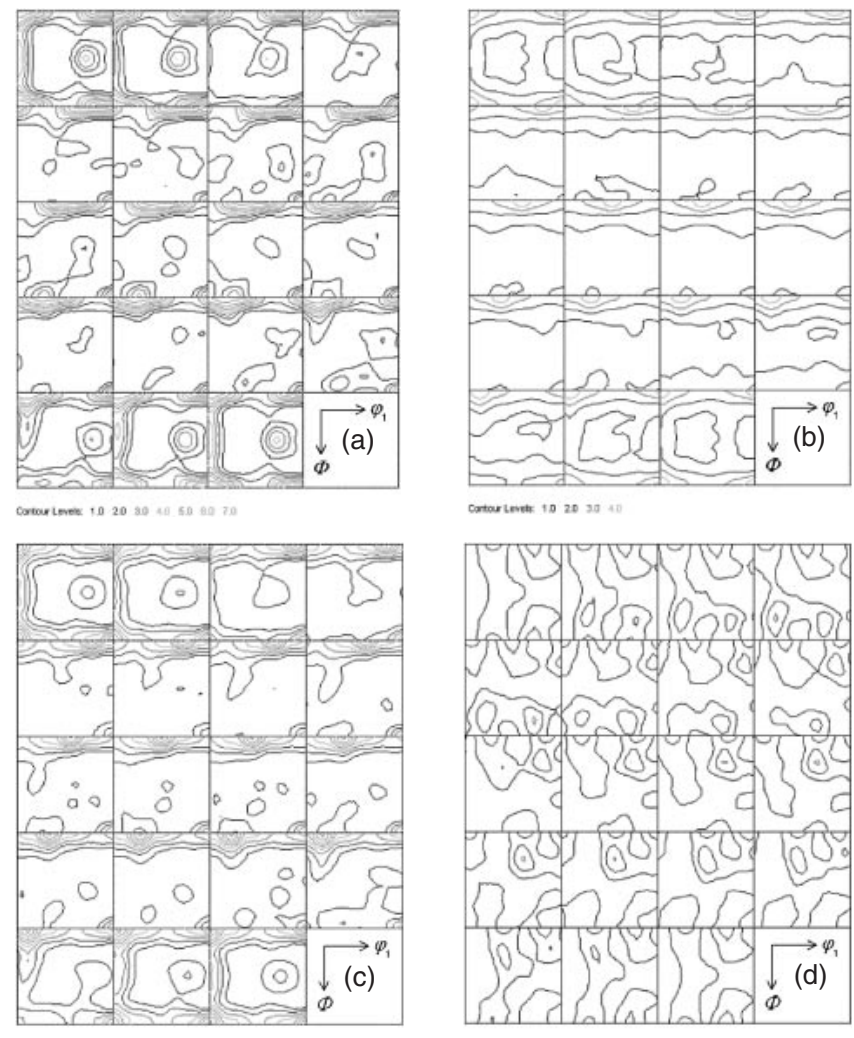

Fig. 10 Overall textures through sheet thickness for T4-treated AA6016 alloy sheets, (a) cold rolled to $90 \%$ reduction or (b) warm rolled to $90 \%$ reduction at $673 \mathrm{~K}$. Partial textures in (c) center and (d) surface layers are also shown for the warm rolled and T4-treated specimen (b) with texture gradient. Contour levels: 1, 2, 3, 4, 5, 6, 7, 8 .
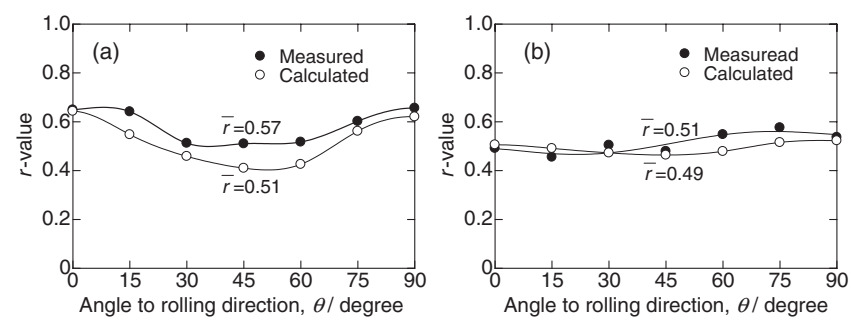

Fig. 11 Planar anisotropy of $r$-value measured and calculated by the full constrains (FC) model from overall textures through sheet thickness for (a) cold rolled or (b) warm rolled and T4-treated samples shown in Fig. 10.

best agreement. This suggests that homogeneous deformation occurred for Al-Mg-Si alloys, differently from Al-Mg alloys. These results are quite consistent with the results of AA5052 and AA6061 without texture gradient in the previous study. ${ }^{5)}$ Overall texture through sheet thickness, thus, leads to good prediction of $r$-value, even though the sheet material has inhomogeneous texture along the thickness direction. When comparing the measured $r$-values between cold and warm rolled samples, the latter shows a lower average $r$-value than the former, unlike the case of Al-Mg alloy sheets. For Al-MgSi alloy sheets, warm rolling seems to be ineffective in improving average $r$-value because of solution treatment at a much higher temperature after warm rolling.

\subsubsection{Change in $\boldsymbol{r}$-value by texture gradient}

Even for samples with texture gradient along the thickness 


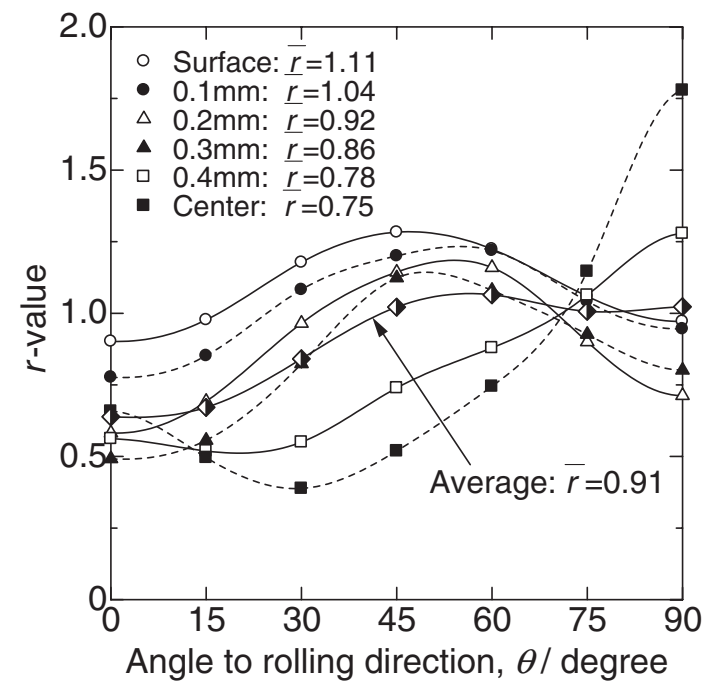

Fig. 12 Planar anisotropy of $r$-value at different locations calculated by the relaxed constraints (RC) model from the texture at each layer for a warm rolled and annealed $\mathrm{Al}-4.3 \% \mathrm{Mg}-0.3 \% \mathrm{Fe}$ alloy sheet (sample A).

direction, the measured $r$-value could be successfully related to overall recrystallization texture through sheet thickness by using appropriate calculation models. It is necessary to reveal what locations in a sample have high $r$-values, which must contribute to improving the $r$-value as a whole in a sheet material, in order to obtain good formability in texturegraded aluminum alloy sheets. Therefore, recrystallization textures at various locations along the thickness direction ware measured for a warm rolled and annealed $\mathrm{Al}-4.3 \% \mathrm{Mg}$ $0.3 \% \mathrm{Fe}$ alloy with inhomogeneous texture. Figure 12 shows planar anisotropy of $r$-value at the various locations calculated from the measured textures by the RC model with relaxed shear strain perpendicular to the tensile direction. The anisotropy of $r$-value and the resultant average $r$-value varies significantly depending on the location. The surface layer indicates the highest average $r$-value of 1.11 and the highest minimum $r$-value of 0.90 at the $0^{\circ}$ direction, while the center layer indicates the lowest average $r$-value of 0.75 and the lowest minimum $r$-value of 0.39 at the $30^{\circ}$ direction. Obviously, these are closely related to the principal texture components that were $\{111\}\langle 110\rangle$ and cube orientations in the surface and center layers, respectively. Surprisingly, the average $r$-value as a whole is 0.91 and almost the same value as a measured value of 0.90 and a calculated value of 0.90 from overall texture through thickness. This reveals that the local layer with a specific texture is unlikely to affect the average $r$-value as a whole in samples with texture gradient. At the same time, it is supposed that the sheet material having higher $r$-values in the surface layer than in the center layer may be more favorable for deep drawing, since at the corner of a punch it undergoes deformation like bending which gives the largest strain on the surface.

Similarly, a warm rolled and T4-treated AA6016 alloy sheet clearly showed a change in texture along the thickness direction, as observed in Fig. 10(c) and (d). Planar anisotropy of $r$-value calculated by the FC model in the surface, intermediate and center layers is shown in Fig. 13. Obviously, the calculated average $r$-value in the surface layer is

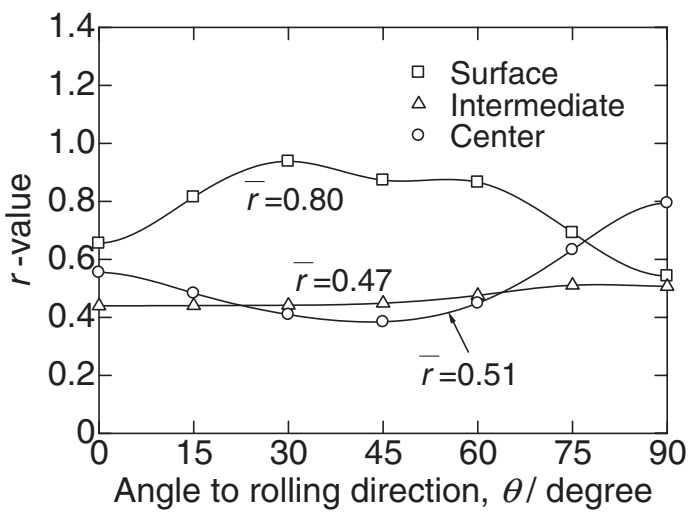

Fig. 13 Planar anisotropy of $r$-value at different locations calculated by the full constraints (FC) model from the texture at each layer for a warm rolled and T4-treated AA6016 alloy sheet.

much higher than the others. This is probably due to the existence of near-R orientation as a main component in the surface layer, which enhances $r$-value around the $45^{\circ}$ direction. However, the average $r$-value of 0.49 calculated from overall texture through thickness shown in Fig. 11(b) is close to calculated average $r$-values in the center and intermediate layers. This suggests that the surface recrystallization texture favorable for improving $r$-value was not deeply developed toward the intermediate layer. Moreover, in spite of the existence of the 'thin' surface texture with much higher average $r$-value, the measured average $r$-value was lower for a warm rolled sample (0.51) than for a cold rolled sample (0.57), as shown in Fig. 11. The center layer of the warm rolled sample had a relatively similar recrystallization texture to all three layers of the cold rolled sample, but it consisted of cube, Goss and weak P orientations. According to calculation by the FC model, P orientation with Gaussian distribution is predicted to have a high $r$-value above 1.0 around the $15^{\circ}$ direction. From a comparison of measured $r$-values between cold and warm rolled samples, it is found that the $r$-values in an angle range of 0 to $15^{\circ}$ are lower for the warm rolled sample than for the cold rolled one. This corresponds to the fact that $\mathrm{P}$ orientation of the warm rolled sample was weaker than that of cold rolled one in both the center and intermediate layers. It is well known that $\mathrm{P}$ orientation is often observed in recrystallization texture of aluminum alloys containing large-sized second phase particles, such as Al-Mg-Si alloys. ${ }^{19)}$ In addition, $\mathrm{P}$ orientation may be formed in deformation zones with larger strain around particles by the particles stimulated nucleation mechanism during annealing. ${ }^{20)}$ Therefore, it is considered that the weaker $\mathrm{P}$ orientation in the warm rolled sample results from the reduction of deformation zones and/or the decrease of stored energy around particles due to warm rolling. Measurement of deformation textures indicated that $\beta$-fiber texture after warm rolling was stronger than that after cold rolling. If the evolution of deformation zones around particles causes an orientation spread from $\beta$-fiber texture, this result suggests reduced deformation zones in the warm rolled sample. It is also possible that warm rolling leads to lower stored energy around particles than cold rolling because of a much higher rolling temperature. The decrease 


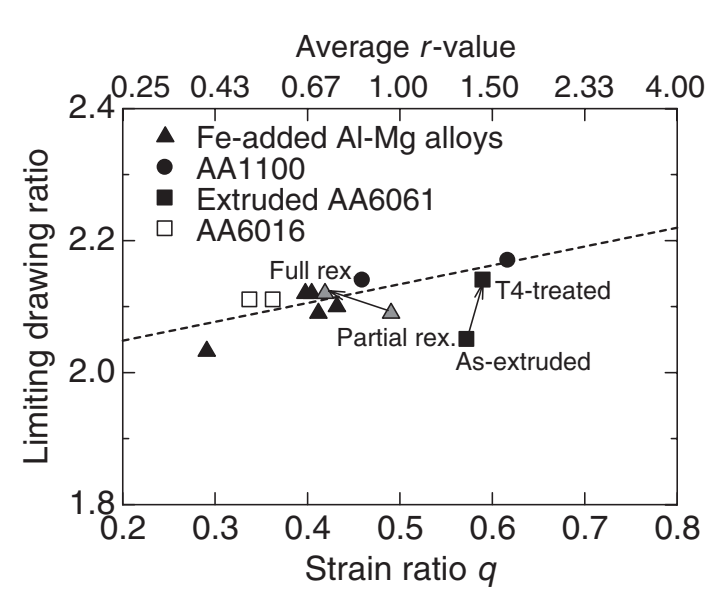

Fig. 14 Correlation between strain ratio of width to length $q$ and limiting drawing ratio (LDR) for aluminum alloys with various textures. All data obtained in the present study are plotted on this graph.

of $\mathrm{P}$ orientation density by such reasons would result in a low average $r$-value in the warm rolled sample, even though the thin surface texture had a high $r$-value. Different rolling processes are required to improve average $r$-value in $\mathrm{Al}-\mathrm{Mg}$ Si alloy sheets. ${ }^{10,21)}$

\section{Discussion}

It has been demonstrated that there is a positive correlation between average $r$-value and LDR in aluminum alloys. However, the correlation in Fig. 6 includes some scatter of experimental data. There is a possibility that average $r$-value is not necessarily an appropriate parameter for representing a correlation with LDR. A change in strain ratio ranging from $r=1$ to $r=0$ is equivalent to that ranging from $r=1$ to $r=\infty$. For example, a change from $r=1$ to $r=0.5$ (a decrement of 0.5 ) corresponds to a change from $r=1$ to $r=2$ (an increment of 1.0). Therefore, a graph with regular intervals of average $r$-value such as Fig. 6 seems to be inappropriate for representing a correlation with LDR. Now we use strain ratio $q^{14,15)}$ instead of $r$-value:

$$
q=-\mathrm{d} \varepsilon_{w} / \mathrm{d} \varepsilon_{l}=r /(1+r)
$$

where $-\mathrm{d} \varepsilon_{w}$ and $\mathrm{d} \varepsilon_{l}$ are true strains in width and in length, respectively. A plot of LDR against strain ratio $q$ is shown in Fig. 14. This graph includes all data measured in the present study. There is a better correlation between $q$ and LDR than between average $r$-value and LDR in comparison with Fig. 6, since LDR increases nearly linearly with increasing $q$-value, except for as-extruded AA6061 and partially recrystallized Fe-added Al-Mg alloy. This probably means that sheet material with higher $q$-value yields a larger contractive strain along the circumferential direction in a flange part under a given tensile strain along the radial direction, and consequently makes it possible to draw the blank with larger diameter. Thus, the use of strain ratio $q$ is more desirable for evaluation of deep drawability. At least, it can be proven from this figure that increasing average $r$-value leads to improvement of deep drawability in aluminum alloys.

LDR is normally evaluated in a fully recrystallized state. The broken line in Fig. 14 is a straight line approximated by the experimental data of fully recrystallized samples. It is possible that deformed or recovered microstructure retained in a sample strongly affects LDR, even if the elongation is apparently sufficient for deep drawing. In order to examine the influence of microstructure on LDR, as hot-extruded AA6061 and partially (80\%) recrystallized Al-Mg-0.8\% Fe alloy were prepared for comparison with T4-treated AA6061 and fully recrystallized $\mathrm{Al}-\mathrm{Mg}-0.8 \% \mathrm{Fe}$ alloy, respectively. It is obvious from Fig. 14 that the as hot-extruded AA6061 has very lower LDR than the T4-treated one in spite of nearly the same $q$-value and that the partially recrystallized Al-Mg alloy has somewhat lower LDR than the fully recrystallized one in spite of a considerably higher $q$-value. These results are attributable to the hot-deformed microstructure with lower ductility and the recovered microstructure with lower ductility that locally remains between recrystallized areas, respectively. A decrease of elongation in local areas causes a more serious problem to LDR than $r$-value. Therefore, LDR must be measured in a fully recrystallized state to clearly show a positive correlation between $q$-value (or average $r$ value) and LDR in aluminum alloys.

\section{Conclusions}

In order to improve deep drawability in aluminum alloys through texture control, recrystallization texture, $r$-value and LDR were investigated using sheet materials prepared by various processes. The chief results obtained in the present study are summarized below.

(1) The artificial sheet specimens with high average $r$ values above 1.4 were prepared by cutting a hot rolled thick plate and a hot extruded bar at appropriate planes that had a $\{111\}$ texture. The use of these specimens permitted an experimental examination of the correlation between average $r$-value and LDR in a wide range. As a result, it was proven that LDR increased with increasing average $r$-value in aluminum alloys. At the same time, there was a better correlation between strain ratio $q$ and LDR than between average $r$-value and LDR.

(2) Warm rolling with large reduction per pass developed shear texture from the sheet surface toward the inside for Fe-added Al-Mg and AA6016 alloys. After annealing, a mixed texture consisting of shear texture components in the surface layer and cube plus $\mathrm{R}$ orientations in the center layer was formed for a $\mathrm{Fe}$ added Al-Mg alloy. This texture led to considerably higher $r$-values at various angles to the rolling direction in the warm rolled sheet than in the cold rolled one. In contrast, a relatively weak cube texture was, on the whole, developed after T4-treatment for an AA6016 alloy, though a different recrystallization texture derived from shear texture was formed near the surface. Such a texture led to a somewhat lower average $r$-value in the warm rolled sheet than in the cold rolled one, in spite of high $r$-value near the surface.

(3) Calculated $r$-values from overall texture through sheet thickness were in good agreement with measured $r$ values in both Fe-added Al-Mg and AA6016 alloys. From these calculations, it is suggested that the RC 
model, which relaxes constraint of shear strain perpendicular to the tensile direction, is suitable for predicting $r$-value in solid-solution hardenable Al-Mg alloys, while the FC model is suitable in age-hardenable Al$\mathrm{Mg}-\mathrm{Si}$ alloys.

\section{Acknowledgements}

This work was supported in part by the project on "Aluminum production and fabrication technology development useful for automotive light-weighting" provided by the New Energy and Industrial Technology Development Organization (NEDO).

\section{REFERENCES}

1) W. T. Lankford, S. C. Snyder and J. A. Bauscher: Trans. Am. Soc. Met. 42 (1950) 1197-1232.

2) Formability of Aluminium Alloy Sheets, ed. by Metal Forming Section, (Japan Institute of Light Metals, Tokyo, 1985).

3) T. Uno: Sumitomo Light Metal Technical Reports 42 (2001) 100-123.

4) G. I. Taylor: J. Inst. Metals 62 (1938) 307-324.

5) H. Inoue and N. Inakazu: J. Japan Inst. Light Met. 44 (1994) 97-103.

6) H. Tanaka, H. Ezaki, K. Yamada, K. Shibue and H. Yoshida: J. Japan Inst. Light Met. 52 (2002) 553-558.
7) T. Kamijo and H. Fukutomi: Proc. 16th Risø Int. Symp. on Materials Science, ed. by N. Hansen et al., (Risø National Laboratory, Roskilde, Denmark, 1995) pp. 377-382.

8) O. Engler, H. C. Kim and M. Y. Huh: Mater. Sci. Technol. 17 (2001) $75-86$.

9) T. Sakai, H. Inagaki and Y. Saito: Proc. 12th Int. Conf. on Textures of Materials, ed. by J. A. Szpunar, (NRC Research Press, Ottawa, 1999) pp. 1142-1147.

10) Y. Miki, K. Koyama, O. Noguchi, Y. Ueno and T. Komatsubara: Mater. Sci. Forum 539-543 (2007) 333-338.

11) M. Dahms and H. J. Bunge: J. Appl. Cryst. 22 (1989) 439-447.

12) H. Inoue and N. Inakazu: J. Japan Inst. Met. 58 (1994) 892-898.

13) H. Inoue and T. Takasugi: Z. Metallkd. 92 (2001) 82-88.

14) H. J. Bunge: Texture Analysis in Materials Science, (Butterworths, London, 1982).

15) H. J. Bunge: Kristall und Technik 5 (1970) 145-175.

16) H. Honneff and H. Mecking: Proc. 5th Int. Conf. on Textures of Materials, ed. by G. Gottstein and K. Lücke, (Springer-Verlag, Berlin, 1978) Vol. I, pp. 265-275.

17) Binary Phase Diagrams, ed. by T. B. Massalski, J. L. Murray, L. H. Bennett and H. Baker, (American Society for Metals, Metals Park, Ohio, 1986) p. 112.

18) R. W. Logan, D. J. Meuleman and W. F. Hosford: Formability and Metallurgical Structure, ed. by A. K. Sachdev and J. D. Embury, (The Metallurgical Society, Warrendale, Pennsylvania, 1987) pp. 159-173.

19) O. Engler and J. Hirsch: Mater. Sci. Eng. A336 (2002) 249-262.

20) O. Engler: Mater. Sci. Technol. 12 (1996) 859-872.

21) H. Inoue, M. Hori, T. Komatsubara, H. Tanaka and T. Takasugi: Mater. Sci. Forum 558-559 (2007) 207-212. 